\title{
A Prospective Randomized Double-blind Comparative Study of Hemodynamic Effects of Etomidate and Propofol in Controlled Hypertensive Patients during Induction of General Anesthesia
}

\author{
Shubha D Bhat ${ }^{1}$, Naina Dalvi ${ }^{2}$, Maithili D Thakur ${ }^{3}$
}

\begin{abstract}
Aim: To study and compare the hemodynamic effects of etomidate and propofol when used in controlled hypertensive patients as an induction agent.

Materials and methods: It is a prospective randomized double-blind comparative study. After Institutional Ethics Committee approval, 60 patients with controlled hypertension undergoing surgery under general anesthesia and willing to participate in the study were included, i.e., the patients fulfilling the inclusion criteria were included.

Results: The hemodynamic parameters like pulse rate, systolic blood pressure (SBP), and diastolic blood pressure (DBP) were well maintained when etomidate was used as an induction agent when compared with propofol. There were no statistically significant side effects like pain on injection, nausea-vomiting, or myoclonus in either of the groups.

Conclusion: IV etomidate $0.3 \mathrm{mg} / \mathrm{kg}$ provides better control of heart rate (HR), SBP, DBP, and mean arterial blood pressure (MAP) during induction, intubation, and after that throughout the procedure when compared with IV propofol $2 \mathrm{mg} / \mathrm{kg}$ in controlled hypertensive patients undergoing general anesthesia.

Clinical significance: Considering the common use of propofol and etomidate as induction agents and the importance of a patient's hemodynamic stability, it is of utmost importance to choose an induction agent carefully in hypertensive patients to prevent more damage to their cardiovascular system.

Keywords: Etomidate, General anesthesia, Injection, Propofol.

Research and Innovation in Anesthesia (2020): 10.5005/jp-journals-10049-0091
\end{abstract}

\section{INTRODUCTION}

Induction of anesthesia is a critical period in hypertensive patients. The deleterious effects of anesthetic agents in such patients are well known. For a hypertensive patient, the occurrence of hypotension, even though blood pressure does not drop to a dramatic level, is a dangerous event for brain, heart, or kidney perfusions. Rapid induction without any serious side effects is a valuable characteristic that is wanted from an ideal induction agent. ${ }^{1}$ Therefore, an appropriate drug should be chosen to maintain hemodynamic stability during induction of anesthesia. Etomidate and propofol are well-known anesthetic agents routinely used for the intravenous induction of anesthesia in hypertensive patients. ${ }^{2,3}$ The two drugs, however, have different induction characteristics. Hence, this study was to compare their effects on hypertensive patients to help choose the better induction agent in this subset of patients.

\section{Materials and Methods}

It was a prospective, double-blind, randomized comparative study. After obtaining Institutional Ethics Committee approval, 60 patients undergoing general anesthesia and willing to participate in the study were included. Inclusion criteria were patients with age 18-60 years, controlled hypertensive patients (ASA II) (BP <140/90 mm $\mathrm{Hg}$ ) taking anti-hypertensive drugs for at least 8 weeks, Mallampati Classification (MPC) grade I and II, and willing to participate in the

\begin{abstract}
${ }^{1-3}$ Department of Anaesthesiology, HBT Medical College and Dr RN Cooper Hospital, Mumbai, Maharashtra, India

Corresponding Author: Naina Dalvi, Department of Anaesthesiology, HBT Medical College and Dr RN Cooper Hospital, Mumbai, Maharashtra, India, Phone: +91 9820711656, e-mail: drnaina@rediffmail.com

How to cite this article: Bhat SD, Dalvi N, Thakur MD, et al. A Prospective Randomized Double-blind Comparative Study of Hemodynamic Effects of Etomidate and Propofol in Controlled Hypertensive Patients during Induction of General Anesthesia. Res Inno in Anesth 2020;5(2):33-38.
\end{abstract}

Source of support: Nil

Conflict of interest: None

study. Unwilling patients, pregnant patients, patients with other comorbidities like ischaemic heart disease (IHD), diabetes mellitus (DM), etc., ASA I, III, IV, MPC > grade II, patients allergic to eggs/ propofol were excluded from the study.

Informed written consent was taken from each patient before scheduled operations. All patients were properly assessed preoperatively. By the computer-generated randomized method, patients were randomized to receive Inj. etomidate (group E) or Inj. propofol (group P). Group E: Inj. etomidate $0.3 \mathrm{mg} / \mathrm{kg}$ and group P: Inj. propofol $2 \mathrm{mg} / \mathrm{kg}$.

(c) The Author(s). 2020 Open Access This article is distributed under the terms of the Creative Commons Attribution 4.0 International License (https:// creativecommons.org/licenses/by-nc/4.0/), which permits unrestricted use, distribution, and non-commercial reproduction in any medium, provided you give appropriate credit to the original author(s) and the source, provide a link to the Creative Commons license, and indicate if changes were made. The Creative Commons Public Domain Dedication waiver (http://creativecommons.org/publicdomain/zero/1.0/) applies to the data made available in this article, unless otherwise stated. 
All patients were subjected to the same premedications with Inj. midazolam $0.03 \mathrm{mg} / \mathrm{kg}$, Inj. fentanyl $2 \mu \mathrm{g} / \mathrm{kg}$, and Inj. glycopyrrolate $4 \mu \mathrm{g} / \mathrm{kg}$. After sedation, vitals were noted. As per randomization, induction was performed using the selected induction agent for each group. Inj. etomidate $0.3 \mathrm{mg} / \mathrm{kg}$ or Inj. propofol $2 \mathrm{mg} / \mathrm{kg}$ as per the above-mentioned groups were given. All patients of both groups were given Inj. loxicard $1.5 \mathrm{mg} /$ $\mathrm{kg} 90$ seconds before induction to alleviate pain during injection. Both groups were given Inj. succinylcholine $2 \mathrm{mg} / \mathrm{kg}$ as a muscle relaxant before intubation which was done by an experienced anesthesiologist. Patients were maintained on oxygen, nitrous oxide, desflurane, and intermittent doses of Inj. vecuronium 0.08 $\mathrm{mg} / \mathrm{kg}$.

Monitoring was done by the investigator who was unaware of the drug given. Patients were monitored every minute for 5 minutes after induction and then every 5 minutes till 30 minutes for changes in the following parameters:

- Heart rate (HR).

- Systolic blood pressure (SBP).

- Diastolic blood pressure (DBP).

- Mean arterial blood pressure (MAP).

Patients were monitored intraoperatively every 15 minutes thereafter for a maximum duration of 90 minutes. The following were the observation timings:

Preoperative vitals.

T0-Baseline vitals after sedation.

T1-1 minute post-induction.

T2-2 minutes post-induction.

T3-at intubation.

T4-1 minute post-intubation.

T5-2 minutes post-intubation.

T10, T15, T20, T25, T30, T45, T60, T75, T90-10, 15, 20, 25, 30, 45, 60,75 , and 90 minutes post-induction, respectively.

Side effects like postoperative nausea vomiting (PONV), myoclonus, pain on injection if any were observed and noted.

After observing and collecting intraoperative and postoperative data of all 60 patients, decoding of the drug was done.

\section{Results}

\section{Demographic Data}

The preoperative HR of the study subjects in group E was $81.87 \pm$ 12.10 and in group $P$ it was $80.90 \pm 9.75$ and this difference was not statistically significant $(p=0.7337)$. In group $E$, the HR decreased steadily from T0 (baseline) to T5 (5 minutes post-induction) and later the HR showed an increasing trend and came back to $80.12 \pm 12.13$ by the end of surgery. In group P, the HR decreased significantly when compared with group E from T2 (2 minutes post-induction) to T25 (25 minutes post-induction) ( $p$ value $<0.05$ ) and later it recovered back to $79.78 \pm 7.23$ at the end of surgery (Tables $1 \mathrm{~A}$ and 1 B and Fig. 1).

The mean preoperative SBP of the study subjects in group $E$ was $132.53 \pm 9.61$ and in group $P$ it was $133.40 \pm 8.70$ which was not significant ( $p$ value $>0.05$ ) when compared with each other. In group $\mathrm{E}$, there was a slight fall in SBP decreased from T0 (baseline after sedation) to T1 (1 minute post-induction) and later was almost around the baseline value till the end of surgery. But, in group $P$, the SBP decreased significantly when compared with group E from T1 (1 minute post-induction) to T3 (at intubation) ( $p$ value $<0.05$ ) but, however, it increased to $123.50 \pm 13.17$ levels at T4 ( 1 minute postintubation). Furthermore, there was again significant fall in SBP from T5 (2 minutes post-induction) to T25 (25 minutes post-induction) in group $\mathrm{P}$ when compared with group $\mathrm{E}$ ( $p$ value $<0.05$ ) (Fig. 2).

The preoperative DBP of the study subjects in group E was 86.60 \pm 8.80 and in group $P$ it was $87.50 \pm 7.17$ which was not statistically significant ( $p$ value $>0.05$ ) when compared with each other. In group E, the DBP decreased from T0 (baseline after sedation) to T3 (at induction) and later increased steadily to $79.23 \pm 12.27$ levels till the end of surgery. In group $P$, the decrease in DBP was significantly lower ( $p$ value $<0.05$ ) from T1 (1 minute post-induction) to T3 (at intubation) and later increased gradually to $80.44 \pm 12.88$ levels at the end of surgery (Fig. 3).

The preoperative mean arterial pressure of the study subjects in group $E$ was $101.91 \pm 9.07$ and in group $P$ it was $102.80 \pm 7.68$ which was not statistically significant when both the groups were compared. In group $E$, the fall in mean arterial pressure was gradual from T0 (baseline after sedation) to T10 (10 minutes postinduction) and later it recovered back to $96.37 \pm 12.29$ by the end of surgery. In group $\mathrm{P}$, the mean arterial pressure significantly reduced throughout from T1 (1 minute after induction) to T3 (at intubation) and later from T5 (2 minutes after intubation) to T15 (15 minutes post-induction) ( $p$ value $<0.05$ ) when compared with group $\mathrm{E}$. Furthermore, the mean arterial pressure increased to $97.40 \pm 12.77$ levels by the end of surgery (Table 2 and Fig. 4).

\section{Discussion}

Hypertensive patients remain a major concern for the anesthesiologists because firstly it is the most common etiology of chronic cardiovascular disease in adult patients who undergo surgery and secondly, despite tremendous improvement in the management of hypertension, many of these patients have not fully controlled hypertension when they present on the day of surgery. The hypotension threshold that is associated with a decrease in blood flow to organs is at a higher blood pressure level in hypertensive patients than in normotensives. For a hypertensive patient, the occurrence of hypotension, even though blood pressure

Table 1A: Age distribution

\begin{tabular}{|c|c|c|c|c|c|}
\hline \multirow[b]{2}{*}{ Age group } & \multicolumn{2}{|c|}{ Group E } & \multicolumn{2}{|c|}{ Group P } & \multirow[b]{2}{*}{$p$ value } \\
\hline & No & (\%) & No & (\%) & \\
\hline $18-30$ & 1 & 3.33 & 2 & 6.67 & 0.4534 \\
\hline $31-40$ & 8 & 26.67 & 6 & 20.00 & \\
\hline $41-50$ & 10 & 33.33 & 12 & 40.00 & \\
\hline $51-60$ & 11 & 36.67 & 10 & 33.33 & \\
\hline Total & 30 & 100 & 30 & 100 & \\
\hline Mean & 51.23 & & 52.23 & & 0.9163 \\
\hline SD & 7.11 & & 7.56 & & \\
\hline
\end{tabular}


Table 1B: Sex distribution

\begin{tabular}{|c|c|c|c|c|c|}
\hline \multirow[b]{2}{*}{ Gender } & \multicolumn{2}{|c|}{ Group E } & \multicolumn{2}{|c|}{ Group P } & \multirow[b]{2}{*}{$p$ value } \\
\hline & No & (\%) & No & (\%) & \\
\hline Female & 24 & 80.00 & 22 & 73.33 & 0.5457 \\
\hline Male & 6 & 20.00 & 8 & 26.67 & \\
\hline Total & 30 & 100 & 30 & 100 & \\
\hline
\end{tabular}

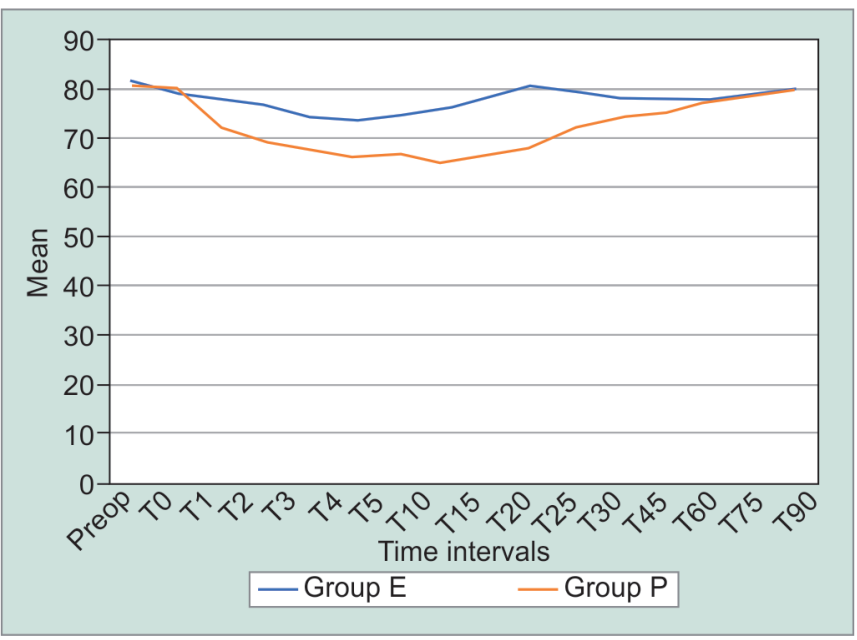

Fig. 1: Effect of study drug on heart rate at different time intervals

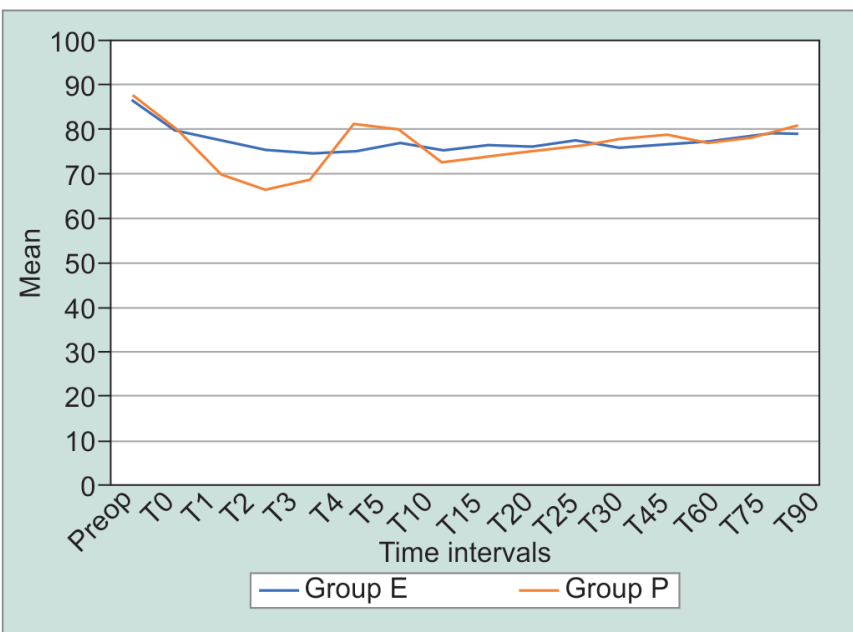

Fig. 3: Effect of study drug on diastolic blood pressure at different time intervals

does not drop to a dramatic level, is a dangerous event for brain, heart, or kidney perfusions.

Induction of anesthesia is a critical period in hypertensive patients. The deleterious effects of anesthetic agents in such patients are well known. Rapid induction without any serious side effects is a valuable characteristic that is wanted from an ideal induction agent. Therefore, an appropriate drug should be chosen to maintain hemodynamic stability during the induction of anesthesia. Etomidate and propofol are well-known anesthetic agents routinely used for the intravenous induction of anesthesia in hypertensive patients. The two drugs, however, have different induction characteristics. ${ }^{4}$ Hence, we are doing this study to compare their effects on hypertensive patients to help choose the better induction agent in this subset of patients.

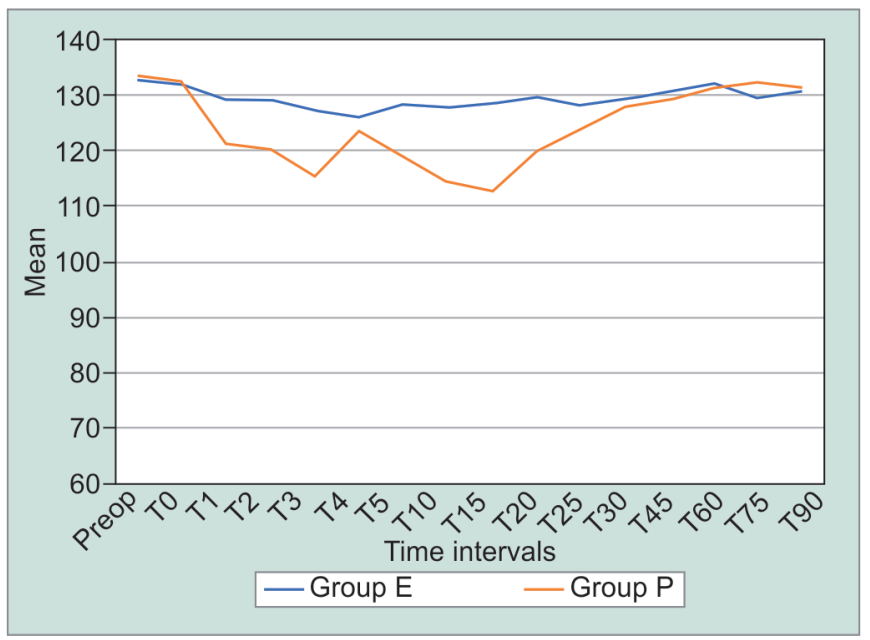

Fig. 2: Effect of study drug on systolic blood pressure at different time intervals

In our study, we have attempted to compare the hemodynamic effects of etomidate and propofol and side effects if any when used as an induction agent in controlled hypertensive patients.

\section{Heart Rate}

In our study, in both the groups, the mean HR decreased after sedation and induction but the fall was statistically significant ( $p$ $<0.05$ ) in group P from T2 (2 minutes after induction) till around T25 (25 minutes after induction). While in group $\mathrm{E}$ too, there was a fall in HR from T0 (baseline after sedation) till T5 (5 minutes after induction) which was not statistically significant $(p>0.05)$. When compared with mean baseline value T0, group E showed no significant decrease while there was a significant decrease in HR in group $\mathrm{P}(p<0.001)$.

Similar results were found by Kumar in $2016^{5}$ and by Kumar et al. in 2018. ${ }^{6}$ In 2015, Das et al. ${ }^{7}$ studied hemodynamic effects of etomidate, propofol, and thiopentone in laparoscopic cholecystectomy surgeries. They found that post-induction and after intubation, in the etomidate group, HR did not significantly change compared with pre-induction. But, in the propofol group, the post-induction HR significantly decreased and after intubation, significantly increased compared with the pre-induction. Similarly, in March 2016, Sharma et al. ${ }^{8}$ showed that the decrease in mean HR in both groups, propofol and etomidate were statistically significant $(p<0.05)$ with exaggerated fall in HR with propofol. Also, Meena et al. in $2016^{1}$ studied 90 patients in 3 groups using induction agents as propofol, etomidate, and etomidate-propofol combination. Heart rate in all the three groups decreased after induction and it was more in the propofol group. In 2018, Patel et al. ${ }^{9}$ observed that HR decreased after induction in all groups, propofol, etomidate, propofol, and etomidate and it was more in patients receiving propofol. Heart rate increased in all groups but more in patients receiving etomidate after intubation. 
Table 2: Distribution of study subjects based on side effects

\begin{tabular}{|c|c|c|c|c|c|}
\hline \multirow[b]{2}{*}{ Side effects } & \multicolumn{2}{|c|}{ Group E } & \multicolumn{2}{|c|}{ Group P } & \multirow[b]{2}{*}{$p$ value } \\
\hline & No & (\%) & No & (\%) & \\
\hline Nausea and vomiting & 2 & 6.67 & 3 & 10.00 & 0.4532 \\
\hline Myoclonus & 0 & 0 & 0 & 0 & - \\
\hline Pain on injection & 3 & 10.00 & 5 & 16.67 & 0.1222 \\
\hline
\end{tabular}

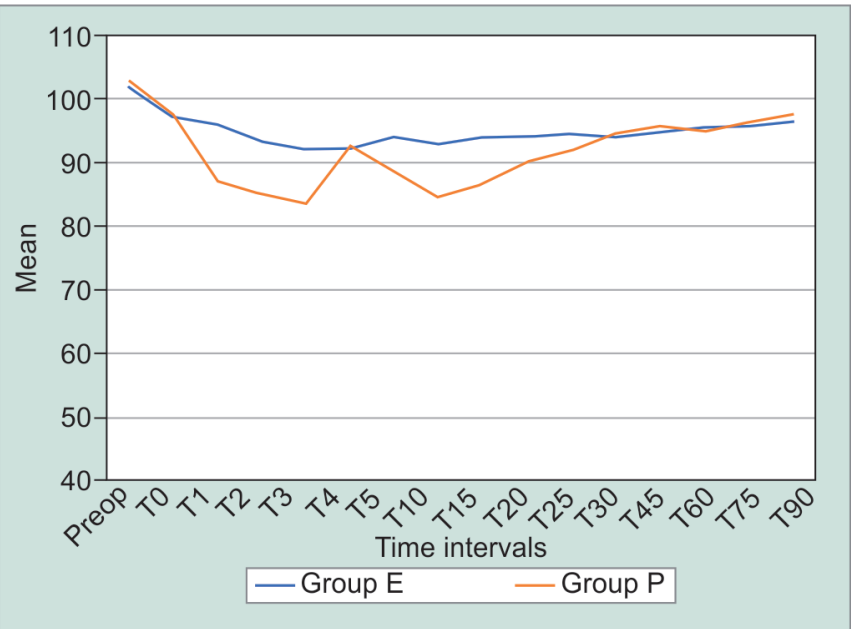

Fig. 4: Effect of study drug on mean arterial blood pressure at different time intervals

In hypertensive patients, it was seen that in 2005 , MalinowskaZaprzalka et al. ${ }^{10}$ while studying hemodynamic changes in enalapril treated hypertensive patients during induction of general anesthesia using propofol and etomidate found that $\mathrm{HR}$ reduced significantly in enalapril treated patients after propofol, but HR increased in case of etomidate. Also, in 2018, Shah et al. ${ }^{11}$ found that post-induction, HR did not change significantly in the etomidate group, but in the propofol group, it decreased significantly compared with the pre-induction value (3.8 vs $6.5 \%$ ) in hypertensive patients.

Thus, in our study, we found results similar to Sharma et al. ${ }^{8}$ and Meena et al. ${ }^{1}$ There was a fall in HR due to both propofol and etomidate induction. But fall due to propofol was statistically significant. Even the intubation response did not cause any increase in HR in both the groups. The fall in HR was not very significant in the etomidate group and the values returned to the baseline earlier than the propofol group. It may be due to the hypotension caused by propofol due to reduction in the heart's preload and afterload is not synchronized with the heart's compensatory responses such as increased cardiac output and increased HR. This hemodynamic drop may be intensified by high doses of the drug and high speed of injection. So, there is subsequent bradycardia due to propofol. Another reason could be the superadded effect of chronic antihypertensive therapy like beta-blockers causing bradycardia during induction of both agents. However, etomidate maintained its hemodynamic stability by maintaining the HR throughout except for the initial fall in HR.

\section{Systolic Blood Pressure}

The mean preoperative SBP between the two groups was not statistically significant till the T0 value (baseline after sedation). Later, there was a fall in both the groups on induction which was statistically significant in group $\mathrm{P}(p<0.05)$ till T25 $(25$ minutes after induction) except at T4 where there was no difference between the two groups ( $p=0.35$ ) as there was intubation pressor response observed in group P. In group E, hemodynamic stability was maintained till the end of surgery.

Kumar et al. in $2018^{6}$ and Muriel et al. in $1991^{12}$ found that postintubation, due to pressor response, there has been increased SBP in both the groups followed by maintained SBP in the etomidate group but persistent hypotension in the propofol group. These results were consistent with our studies. They found that intravenous injection of propofol and etomidate was followed by a decrease in SBP. After tracheal intubation, there was a significant increase in SBP in the etomidate group. These changes were minimal after giving propofol. In 2016, Sharma et al. ${ }^{8}$ in their study observed that decrease in SBP in propofol induced patients was statistically significant $(p<0.02)$ compared with the decrease in SBP in patients treated with etomidate and remained significant even up to 10 minutes post-intubation.

In hypertensive patients, in 2005, Malinowska-Zaprzalka et al. ${ }^{10}$ studied hemodynamic changes in enalapril treated hypertensive patients during induction of general anesthesia using propofol and etomidate and found that SBP was significantly lower in patients treated with enalapril when given propofol than hypertensive or healthy patients given etomidate. In a study similar to ours, Shah et al. in $2018^{11}$ found that there was no significant change in the etomidate group during the post-induction period, but in the propofol group, mean SBP decreased significantly after induction $(p<0.0001)$.

Thus, in our study, we observed a fall in SBP in both the groups, propofol and etomidate but, the mean fall in group $P$ was significant than that in group E. Post-intubation, due to pressor response, there has been increased SBP in both the groups followed by maintained SBP in etomidate group but persistent hypotension in the propofol group. The reason for this could be attributed to the fact that propofol-induced hypotension is mediated by an inhibition of the sympathetic nervous system and impairment of baroreflex regulatory mechanisms. Conversely, etomidate maintained hemodynamic stability through the preservation of both sympathetic outflow and autonomic reflexes and baroreceptor functions ${ }^{10}$ and capacity to bind and stimulate peripheral alpha-2B adrenergic receptors with subsequent vasoconstriction. A decrease in systemic blood pressure after bolus injection of propofol is dependent on both vasodilation with reduced preload and afterload and myocardial depression (negative inotropic action).

Laryngoscopy and tracheal intubation, common maneuvers in anesthetic procedures, can be associated with an increase in $\mathrm{HR}$ and blood pressure secondary to the sympathetic stimulation. Data in the literature indicate a more pronounced hemodynamic response in hypertensive patients than in normotensive individuals secondary to adaptive cardiovascular changes and sympathetic hyperactivity. On the contrary, in the present study, a 
greater percentage of elevations in blood pressure in hypertensive patients than in normotensive individuals were not observed. A possible explanation is that only ASA II hypertensive patients, and most of them with the diagnosis of stage $1(71.8 \%)$ hypertension, were included; SBP $>180 \mathrm{~mm} \mathrm{Hg}$ and diastolic pressure $>110 \mathrm{~mm}$ $\mathrm{Hg}$ are associated with a greater incidence of cardiovascular lability.

Hypertensive patients especially those who are controlled on more than two drugs are more prone to hypotension on induction because their baroreceptor slopes are set at higher levels.

\section{Diastolic Blood Pressure}

In our study, the mean preoperative DBP between the two groups was not statistically significant till the T0 value (baseline after sedation). Later, there was a fall in both the groups on induction which was statistically significant in group P $(p<0.05)$ till T3 (at intubation). There was no difference between the two groups ( $p>$ 0.05) from there onward till the end of surgery.

Kumar et al. in $2018^{6}$ found that intravenous injection of propofol and etomidate was followed by a decrease in diastolic arterial pressure. After tracheal intubation, there was a significant increase in diastolic arterial pressure in the etomidate group. These changes were minimal after giving propofol. In 2015, Das et al. ${ }^{7}$ studied hemodynamic effects of etomidate, propofol, and thiopentone in laparoscopic cholecystectomy surgeries. They found that in the etomidate group, post-induction and after intubation, DBP did not change significantly. But, in propofol and thiopentone groups, DBP decreased after induction and again increased after intubation. However, in 2016, Sharma et al. ${ }^{8}$ observed in their study that decrease in DBP in both the group was statistically significant $(p<0.05)$ for basal, post-intubation at 5 and 10 minutes.

In hypertensive patients, in 2005, Malinowska-Zaprzalka et al. ${ }^{10}$ studied hemodynamic changes in enalapril treated hypertensive patients during induction of general anesthesia using propofol and etomidate and found that DBP was significantly lower in patients treated with enalapril when given propofol than hypertensive or healthy patients given etomidate. Shah et al. in $2018^{11}$ found that there was a significant decrease in the propofol group $(p<0.0001)$ but mean DBP did not decrease significantly in the etomidate group. In cardiac patients, Pandey in 2012 found a significant fall in DBP with propofol when compared with etomidate in patients with normal LV function undergoing CABG on CPB. Similarly, Kaur et al.in $2014^{13}$ found that the mean DBP measured before induction was stable and comparable in two groups $(p>0.05)$.

In our study population, we did not observe a greater percentage reduction in DBP in hypertensive patients when compared with normotensive patients, probably due to vascular remodeling and increased sympathetic tonus by compensatory mechanisms caused directly by hypertension, which are responsible for the maintenance of an elevated systemic vascular resistance.

\section{Mean Arterial Pressure}

In our study, there was no difference between the mean MAP of the two groups preoperatively and at TO (baseline after sedation). Furthermore, there was a decrease in MAP in both the groups but it was statistically significant $(p<0.05)$ in group $\mathrm{P}$ when compared with group $\mathrm{E}$ till 15 minutes after induction
(T15) except at T4 (1 minute post-intubation) where there was no significant difference statistically owing to the pressor response in group $P$.

When compared with the baseline values of both the groups (T0), there was a statistically significant difference in MAP in group $\mathrm{P}(p<0.001)$ except at T4 (1 minute post-intubation) whereas the difference was not significant in group $E$. Thus, hemodynamic stability was maintained in group $\mathrm{E}$.

In 2015, Shah et al. ${ }^{3}$ found that at the 1st and 3rd minute after induction, there was a fall in MAP in both the groups. The fall in MAP was much sharper for the propofol group (24.3 and $28.66 \%$ ) when compared with the etomidate group (15.87 and 16.6\%). The stimulus of laryngoscopy and intubation failed to bring the MAP above baseline levels in the propofol group (3.2\% below baseline) while in the etomidate group there was a $6.9 \%$ rise in MAP above baseline after laryngoscopy. Kumar et al. in $2018^{6}$ found that MAP decreased in both the groups except immediately after intubation where it increased in both the groups because of intubation stress response which was noted minimum when compared with baseline parameters $(p=0.134)$ in the etomidate group. The fall in MAP was more in the propofol group when compared with the etomidate group at 1,3, and 5 minutes after intubation and the difference was statistically significant $(p<0.001)$ but after 15 minutes of intubation, it was comparable in both the groups.

In hypertensive patients, Shah et al. in $2018^{11}$ found that in the etomidate group, post-induction MAP did not decrease, but in the propofol group, it decreased significantly $(p<0.0001)$ in controlled hypertensives. Singh et al. ${ }^{14}$ in 2010 while studying the various induction agents in patients with CAD and left ventricular dysfunction found that there was a significant decrease from the baseline in mean arterial pressure in both propofol and etomidate and also there was an increase in MAP above baseline 1 minute after intubation. The etomidate group was the least effective in minimizing stress response, with a statistically significant increase from baseline in mean arterial pressure $(p=0.001)$ at 1 minute after intubation. Shivanna et al. in $2015^{15}$ while comparing the hemodynamic effects of propofol and etomidate in coronary artery surgeries found that after induction, mean MAP reduced by $30 \%$ in group $P$ and $22 \%$ in group $E$. Reduction was more in group $P$, though not significant statistically. After intubation, the mean MAP continued to be less than the baseline mean MAP. However, it had significantly increased in both groups compared with postinduction levels. In 2015, Kaushal et al. ${ }^{4}$ studied etomidate and propofol induction in cardiac surgeries and found that there was a significant decrease $(p<0.001)$ in MAP between the groups after induction, intubation, and 5 minutes post-intubation. In 2016, Kamath et al. ${ }^{16}$ found following induction and the following intubation, there was no vast difference in MAP between the two groups. Five minutes post-intubation, there was an increase in MAP in the propofol group which was statistically significant. Thus, in our study, we found that there was a fall in MAP in both groups after induction. Also, the fall in MAP post-induction was much more and significant for the propofol group when compared with the etomidate group. Larsen et al. ${ }^{17}$ examined the effects of propofol upon myocardial function by measuring changes in left ventricle function using transthoracic tissue-Doppler echocardiography and concluded that a decrease in MAP with propofol is secondary to reduce a cardiac filling or a consequence of a direct negative inotropic action of propofol. 
There was no statistically significant difference in both $\mathrm{EtCO}_{2}$ and $\mathrm{SpO}_{2}$ between both the groups throughout the procedure. There were no statistically significant side effects like pain on injection, nausea-vomiting, or myoclonus in either of the groups. Pain on injection was blunted with the help of IV lignocaine 1.5 $\mathrm{mg} / \mathrm{kg}$ administered 90 seconds before induction. Adrenocortical insufficiency which is a known side effect of etomidate could not be studied as serum cortisol levels could not be sent.

The limitation to our study was patients were controlled on a different group of antihypertensive agents and patients with anticipated difficult intubation (MPC grade $>$ II) were not included in the study.

\section{Conclusion}

From our study, we conclude that IV etomidate @0.3 mg/ $\mathrm{kg}$ was found to provide better control of HR, SBP, DBP, and MAP during induction, intubation, and after that throughout the procedure when compared with IV propofol @2 mg/kg in controlled hypertensive patients undergoing general anesthesia. Etomidate is found to be a near-ideal induction agent in patients with hypertension and even in those with cardiac diseases and can continue to be the induction agent of choice in the future too.

\section{Clinical Significance}

Considering the common use of propofol and etomidate as induction agents and the importance of a patient's hemodynamic stability, it is of utmost importance to choose an induction agent carefully in hypertensive patients to prevent more damage to their cardiovascular system.

\section{References}

1. Meena K, Meena R, Nayak SS, et al. A comparative study of effect of propofol, etomidate and propofol plus etomidate induction on hemodynamic response to endotracheal intubation: a RCT. J Anesth Clin Res 2016;7(5):1000622. DOI: 10.4172/2155-6148.1000622.

2. Lunia A, Khilji MY, Rehman $\mathrm{H}$, et al. A randomised controlled trial to compare etomidate and propofol for induction of general anesthesia in adults. Indian J Basic Appl Med Res 2016;6(1):769-777.

3. Shah S, Chowdhury I, Bhargava A, et al. Comparison of hemodynamic effects of intravenous etomidate versus propofol during induction and intubation using entropy guided hypnosis levels. J Anaesthesiol Clin Pharmacol 2015;31(2):180-185. DOI: 10.4103/0970-9185.155145.
4. Kaushal RP, Vatal A, Pathak R. Effect of etomidate and propofol induction on hemodynamic and endocrine response in patients undergoing coronary artery bypass grafting/mitral valve and aortic valve replacement surgery on cardiopulmonary bypass. Ann Card Anaesth 2015;18(2):172-178. DOI: 10.4103/0971-9784.154470.

5. Kumar Y. Comparison of propofol and etomidate in patients under general anaesthesia. Int J Contempo Med Res 2016;3(12):3488-3490.

6. Kumar A, Shekhawat KK, Sharma R, et al. A comparison of propofol and etomidate as anaesthetic agents for elective non-cardiac surgery. Int J Res Med Sci 2018;6(10):3454-3460. DOI: 10.18203/2320-6012. ijrms20184062.

7. Das M, Pradhan BK, Samantray RC. Comparative study on haemodynamic responses during intubation using etomidate, propofol and thiopentone in laparoscopic cholecystectomy surgeries. Int J Med Health Sci 2015;5(4):150-158.

8. Sharma A, Kulkarni K, Namazi IJ, et al. Comparative study of etomidate-lipuro and propofol for induction in general anaesthesia. Indian J Basic Appl Med Res 2016;5(2):816-823.

9. Patel H, Patel B, Shah D, et al. A comparative study of effect of propofol, etomidate and propofol plus etomidate induction on hemodynamic response to laryngoscopy and endotracheal intubation. Int J Sci Res (IJSR) 2018;7(2):320-324.

10. Malinowska-Zaprzalka M, Wojewodzka M, Dryl D, et al. Hemodynamic effect of propofol in enalapril treated hypertensive patients during induction of general anesthesia. Pharmacolog Rep 2005;57:675-678.

11. Shah J, Patel I, Guha A. Comparative study of propofol vs etomidate as an induction agent to evaluate hemodynamic changes during induction of anesthesia in controlled hypertensive patients. Anaesth Pain Intensive Care 2018;22(3):361-367.

12. Muriel C, Santos J, Espinel C. Comparative study of propofol with thiopental and etomidate in anesthetic induction. Rev Esp Anestesiol Reanim 1991;38(5):301-304.

13. Kaur S, Kataria AP, Kaur G, et al. Comparison of induction characteristics of propofol-lipuro and etomidate-lipuro in cardiac patients in noncardiac surgery. Int J Sci Stud 2014;2(6):66-72.

14. Choudhury $M$, Kapoor $P$, Kiran $U$, et al. A randomized trial of anaesthetic induction agents in patients with coronary artery disease and left ventricular dysfunction. Ann Card Anaesth 2010;13(3):217223. DOI: $10.4103 / 0971-9784.69057$

15. Shivanna S, Priye $S$, Jagannath $S$, et al. A comparative study of haemodynamic effects of propofol and etomidate as an induction agent in coronary artery surgery. J Evolut Med Dent Sci 2015;4(4):598607. DOI: 10.14260/jemds/2015/88.

16. Kamath MR, Kamath S, Patla KP. Propofol or etomidate: does it genuinely matter for induction in cardiac surgical procedures? Indian J Clin Anaesthe 2016;3(4):551-555.

17. Larsen JR, Torp P, Norrild K, et al. Propofol reduces tissue-Doppler markers of left ventricle function: $A$ transthoracic echocardiographic study. Br J Anaesth 2007;98:183-188 